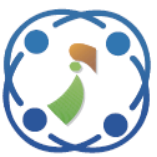

\title{
Hybrid Quantum Salp Swarm Algorithm for Contrast Enhancement of Natural Images
}

\author{
Gehad Ismail Sayed $^{1 *} \quad$ Ghada Khoriba $^{2} \quad$ Mohamed H. Haggag $^{2}$ \\ ${ }^{1}$ Faculty of Computers and Information, Cairo University, Egypt \\ ${ }^{2}$ Faculty of Computers and Information, Helwan University, Egypt \\ * Corresponding author's Email: GehadIsmail_FCI@yahoo.com
}

\begin{abstract}
This paper proposes a new hybrid approach based on quantum computing and SSA to boost the performance of SSA through finding the optimal trade-off between exploitation and exploration. QSSA relies on embedding the integration of the quantum operators and interference in the optimization process of SSA and the quantum representation of the search space. QSSA is tested on 29 global benchmark functions of CEC2017 and 8 benchmark images. The simulation results showed that employing the principles of quantum can significantly boost the performance of SSA. The performance of QSSA is compared with SSA and other recent and well-known optimization algorithms. The results on CEC2017 demonstrated the capability of QSSA to find the global optima for most of unimodal and multimodal benchmark functions, especially with complex search space. Moreover, the results revealed that the proposed image enhancement based QSSA is robust and can distinctly enhance the contrast of the color images.
\end{abstract}

Keywords: Quantum theory, Salp swarm algorithm, Contrast enhancement, Global optimization.

\section{Introduction}

Optimization has aroused a great interest in many disciplines of science and engineering. It is a process with the aim to search for the best solution among all available ones of a particular problem. In consideration of the optimization algorithms, there are two categories that can be employed to solve optimization problems: deterministic or conventional algorithms and meta-heuristics intelligent algorithms [1]. The conventional algorithms such as gradient-based algorithm, Newton and quasi Newton methods, steepest descent method, and sequential quadratic programming are being used to solve global optimization problems. Unfortunately, the associated predefined assumptions of these methods make them inefficient and inaccurate enough in solving today's complex optimization problems characterized by being non-continuous, nondifferential, and multimodal aspects. In contradiction to conventional algorithms, metaheuristic intelligent algorithms (MIAs) have been developed in order to overcome the restrictions of the conventional algorithms. The first advantage of the MIAs is that they are flexible and easy to deal with different types of problems. The second one is that they don't need gradient information when solving optimization problems. Third, their intrinsic exploration and exploitation benefits decrease the probability of sucking in local optima.

Most of the MIAs are inspired by nature behaviors, animal behavior or physical phenomena. In this context, the MIAs are classified into three main categories: swarm-based algorithms, evolutionary based and physics-based. Evolutionary based algorithms perform the optimization process by mimicking the evolution scenario in the nature [2], while physics-based methods imitate some rules of physics in the universe. While, the third category represents the swarm-based techniques, simulates the behavior of animals that foraging in groups. Although MIAs have introduced satisfactory 
outcomes in solving optimization problems, the performance of these algorithms are affected by their algorithm-specific or introduced parameters. Thus, setting appropriate parameters for the candidate problem represents a difficult task for the stakeholders and the inconvenient setting of parameters can increase the computational cost or attain local solution.

SSA, is one of recent meta-heuristic algorithm proposed by Mirjalili et al. [3] in 2017 for solving optimization problems. It is inspired by the swarming behavior of salps through forming the salp chain. This behavior has the ability to avoid the stagnation in the local optima stagnation to some extent. However, it cannot always perform the searching process well. Thus, SSA fails to obtain a global solution in some cases. Hence, SSA lacks to strategy to improve the best so far solution, the quality of obtained solutions may not be good enough.

Quantum computing $(\mathrm{QC})$ is a new research area inspired by principles of quantum mechanics that was developed by Benioff and Eeynman [4]. The main advantage of QC is that, the ability of simultaneous processing huge numbers of quantum states in parallel. In this side, QC exhibits new philosophy in optimization field due to its interesting principles. Thus, growing interests have been devoted by many research papers on incorporating classical evolutionary algorithms and quantum computing [5, 6]. By this incorporation, solution efficiency and convergence speed can be improved. Some optimization algorithms based on quantum strategy are introduced, such as quantum genetic algorithm [5], quantum genetic firefly algorithm [7], quantum differential algorithm [8], etc. In this study, a new hybrid approach based on SSA and quantum theory is presented to overcome these problems, namely quantum salp swarm algorithm (QSSA). The proposed hybrid algorithm integrates the quantum computing's main features and salp swarm algorithm in a new one. The proposed QSSA employees the quantum operators such as interference in the optimization process of SSA. The purpose of this hybridization is to obtain better results than the basic SSA by constructing a new update the position of salp, which have a good balancing between exploration and exploitation.

Contrast enhancement (CE) is a very important process that aims to enhance the quality of images and facilitates the operations either detection or recognition. In many applications, the demand is to provide good quality in images [9]. The core objective of $\mathrm{CE}$ is to refine the perception of features of the candidate images which frequently discussed in many areas such as image/video and medical image processing [10]. In this sense, several contrast enhancement approaches have been developed such as histogram equalization [11], contrast limited adaptive histogram equalization [12], and other histogram equalization based algorithms [13, 14]. The traditional contrast enhancement methods (CEMs) do not enough efficient in providing a satisfactory quality of images for some applications such as night vision and satellite imagery. To obtain efficient quality, metaheuristic algorithms are incorporated with traditional CEMs. Getting a generic optimal contrast enhancement algorithm to be applied to all kinds of images is still an open challenge.

In this study, the proposed QSSA is applied to solve two optimization problems. These problems are global optimization and image contrast enhancement problems. A new contrast enhancement approach based on using QSSA and histogram equalization is proposed. The image contrast and intensity values of the image are adjusted by the proposed quantum salp swarm algorithm based image enhancement (QSSAIE). It should be mentioned that there is little in the literature considered to boost SSA's performance [15]. To the best of our knowledge, it is the first time that a quantum version of SSA is proposed. Also, it is the first time that image contrast enhancement is considered in the literature. We can summarize the main contributions of this study as follows:

- This paper considered a new hybrid approach based on quantum theory and SSA.

- The performance of the proposed QSSA algorithm is evaluated on recent global benchmark optimization problems called CEC 2017.

- An improved version of both QSSA and SSA is proposed for image contrast enhancement, where eight well-known benchmark images are adopted.

- Furthermore, the performance of QSSA is compared with the original SSA and other metaheuristic algorithms for both optimization problems.

- As a part of the evaluation process, a set of assessment criteria are utilized. These criteria are; p-estimations of the Wilcoxon rank sum test, mean, standard deviation, mean intensity of the image, peak-to signal ratio and mean square error. 
The remainder of the paper is structured as follow. Section 2 introduces a brief description of the original SSA and quantum computing. In section 3, the novel Quantum Salp Swarm Algorithm (QSSA) is introduced. Section 4 provides the simulation results and discussions. Finally, conclusions and future work are introduced in section 5 .

\section{Basics and background}

\subsection{Quantum computing}

The QC is a relatively new strategy which has flourished as a result of combing the quantum mechanics concepts and computer science. In this regard, the research directions focus on combining the QC strategy with other classical methods through two aspects [16]. The first aspect is to develop quantum algorithms in the classical computers, while the second aspect is to provide the quantum concept into classical algorithms to modify their conventional performances. The basic concepts and laws of quantum mechanics use some phenomena such as entanglement, state superposition and quantum gate [16]. The qubit represents the smallest information unit that can be stored in the two-state quantum computer. Different from the classical bit that employ two possible values only, either 1 or 0 , the qubit appears in the superposition of those values, simultaneously. The state of a qubit may be formulated as a combination of $|0\rangle$ and $|1\rangle$ as follows:

$$
|\Psi\rangle=\alpha|1\rangle+\beta|0\rangle
$$

where $\psi$ denotes state of a qubit which can be represented in the matrix form, $|\psi\rangle=\left[\begin{array}{ll}\alpha, & \beta\end{array}\right]^{T}$, $|0\rangle$ and $|1\rangle$ denote the classical bit values 1 and 0 respectively, whereas $\beta$ and $\alpha$ are complex numbers such that:

$$
\left|\alpha^{2}\right|+\left|\beta^{2}\right|=1
$$

where $\alpha^{2}$ and $\beta^{2}$ represent the probabilities associated with the state"0" and"1", respectively. A register of n-qubits can be contained up to $2 \mathrm{n}$ values at the same time. Consequently, the main feature of the QC is contained in the evolution induced by the linear combination of the basis states $|0\rangle$ and $|1\rangle$. This is known as quantum parallelism [17]. Therefore, quantum algorithms are validated, where they perform well than classical algorithm on several problems [19].

\subsection{Salp swarm algorithm}

The SSA was proposed by Mirjalili et al. [3] to solve optimization problems, where they have modeled the behavior of the salp chain by splitting the population into two classes: leader and followers. The leader represents the salp at the beginning of the chain, whereas the remainder salps are considered as followers. The leader is responsible for guiding the swarm, while the followers make use of each other. Like the other optimization algorithms, the position of each salp is defined in $n$-dimensional space of searching. Therefore, the salps positions are stored in two dimensional matrix called $x l$. It is assumed that food source is considered as the target of the swarm and donated by $F L$. Thus, the leader's position is updated as follows.

$$
x l_{j}^{1}= \begin{cases}F L_{j}+y_{1}\left(\left(U_{j}-L_{j}\right) y_{2}+L_{j}\right), & y_{3} \geq 0 \\ F L_{j}-y_{1}\left(\left(U_{j}-L_{j}\right) y_{2}+L_{j}\right), & y_{3}<0\end{cases}
$$

where $x l_{j}^{1}$ is the position of the leader salp in the $j-t h$ dimension, whereas $F L_{j}$ shows the food source location in the $j-t h$ dimension, the upper and lower bounds of $j-t h$ dimension are denoted by $U_{j}$ and $L_{j}$, respectively and the parameters $y_{2}$ and $y_{3}$ are generated in the interval of $[0,1]$ randomly. The parameter $y_{1}$ is most effective one in SSA because it makes the exploration and exploitation searches in balanced state that is defined as follows:

$$
y_{1}=2 e^{-\left(\frac{4 t}{T}\right)^{2}}
$$

where $t$ denotes the current iteration whereas the maximum number of iterations is denoted by $T$. The position of each follower salp is updated by the following equation:

$$
x f_{j}^{i}=\frac{1}{2} \alpha t^{2}+v_{0} t
$$

where $i \geq 2$ and $x f_{j}^{i}$ denotes the position in $j-t h$ dimension of $i$-th follower salp, $t$ shows the time (Iteration), $v_{0}$ represents the initial velocity, and $\alpha=\frac{v_{\text {final }}}{v_{0}}$ where $v_{\text {final }}=\frac{x l-x l_{0}}{t}$. The discrepancy between iterations is 1 , and by considering $v_{0}=0$, then this equation can be formulated as follows:

$$
x f_{j}^{i}=\frac{1}{2}\left(x f_{j}^{i}+x f_{j}^{i+1}\right), \quad i \geq 2: N
$$


According to Eq. (3) and (6), the salp chain can be simulated.

\section{The novel quantum salp swarm algorithm}

According to Newtonian mechanic's, any particle or individual is described by both its velocity vector $X$ and its position vector $Z$, where $X$ and $\mathrm{Z}$ are used to identify its trajectory, however, the trajectory of a particle doesn't not exist in quantum physics. The reason behind this is that either $\mathrm{Z}$ or $\mathrm{X}$ of the particle cannot be simultaneously determined based on Heisenberg's uncertainty principle. Thus, the behaviour of an algorithm will be changed if one of its particles has quantum behaviour. In this context, quantum salp swarm optimization (QSSA), where each salp has quantum behaviour is motivated from related work proposed in [18-20]. In the proposed QSSA, the dynamic attitude of SSA differs from its original version. Each position of a salp has a state. This state is depicted by probability density function $|\varphi(Z, t)|^{2}$ and wave function $\varphi(Z, t)$. The mathematical representation of the updating position of QSSA is defined as follows:

$$
V(Z)=-\lambda \delta(Z)
$$

where $\lambda$ denotes for constant positive value and $\delta(Z)$ denotes Dirac delta function. Eq. (8) defines the universe wave function in delta potential.

$$
\psi(Z)=\sqrt{\frac{n \lambda}{p^{2}}}
$$

where $p$ is the reduced plank constant, $\mathrm{n}$ is the mass of the salp. We refer to [21] for additional information of the derived equations of wave function. Based on Monte Carlo method, each individual is defined according to Eq. (9) [22]

$$
\begin{aligned}
& Z_{i}(t+1) \\
& = \begin{cases}\alpha-\beta \cdot \mid \text { Mbest }_{i}-Z_{i}(t) \mid \cdot \ln \left(\frac{r}{u}\right), & l< \\
\alpha+\beta \cdot \mid \text { Mbest }_{i}-Z_{i}(t) \mid \cdot \ln \left(\frac{r}{u}\right), & l \geq\end{cases}
\end{aligned}
$$

where $r$ and $u$ are generated randomly from interval $[0,1], \beta$ is called as the contraction-expansion coefficient. $\beta$ is gradually decreased through the curse of iterations. It is mathematically defined at Eq. (10).

$$
\beta=\frac{0.5 \times\left(\operatorname{Max}_{t}-t\right)}{t+0.5}
$$

where $t$ is the iteration number and $\operatorname{Max}_{t}$ is the maximum number of iterations. Mbest denotes the mean best or global point of the population. The mathematical formula is defined at Eq. (11).

$$
\text { Mbest }=\frac{1}{N} \sum_{d=1}^{\text {MaxDim }} \alpha_{b, d}(t)
$$

where $N$ is the population size, $b$ is the optimal salp position, $d$ is the number of dimensions $\alpha$ denotes for the index among the whole salps in the search space and MaxDim is the maximum number of dimensions. The local attractor through this is guaranteed to converge. Eq. (12) shows the mathematical formula.

$$
\alpha=\frac{r_{1} \alpha_{i, d}+r_{2} \alpha_{g, d}}{r_{1}+r_{2}}
$$

where $r_{1}$ and $r_{2}$ are two random numbers generated from uniform distribution in range [0,1], $\alpha_{i, d}$ is the index of $i$-th salp at $d$-th dimension $\alpha_{g, d}$ is the index of $g$-th best salp position at $d$-th dimension.

In this work, the proposed QSSA is applied for contrast enhancement of the images. In QSSA for contrast enhancement, the algorithm starts with setting the maximum number of iterations, population size, number of decision variables (dimension) and the decision boundary. Then, it randomly initialized the positions of salps with random number generated within $[0,255]$ and the length of position vector equals to 256. Then, through the optimization process, each salp position is evaluated using predefined fitness function $F(n)$. In our study, the used fitness function is defined as follows:

$$
F(n)=P_{1}+\lambda \times P_{2}+\gamma \times P_{3}
$$

where $\lambda$ is the contrast scale parameter and $\gamma$ is the amount of details in the image to be retained. Usually, $\lambda$ scaled within $[0,20]$ and $\gamma$ scaled from $[1$, $\left.10^{9}\right]$. In this paper, we set $\lambda$ to 2 and $\gamma$ to 1000 .

$$
P_{1}=\sum(X(t)-H)^{2}
$$

where $H$ is the histogram of the image and $X(t)$ is the position of the salp. 


$$
P_{2}=(X(t)-B)^{2}
$$

where $B$ is the bins location of the histogram.

$$
P_{3}=(X(t) \times D)^{2}
$$

where $D$ is the bi-diagonal difference matrix.

In each iteration, the position of salps is updated. Then, the best position of salp with the best fitness value are updated according to the salp position with the lowest $F(n)$. The optimization process is repeated over and over until the maximum number of iterations is met. Finally, the algorithm terminates and the optimal $\mathrm{Hi}$ is produced. The overall flowchart for the proposed QSSA for contrast enhancement is presented in Fig. 1.

\section{Simulation results and discussions}

In this section, the performance of the proposed QSSA is evaluated to solve two optimization problems. These optimization problems are global optimization problems and contrast enhancement problem. Also, the performance of QSSA is compared with other meta-heuristics algorithms and the classical SSA. Two main experiments were conducted to evaluate the performance of QSSA. The first experiment presented in Section 4.1 aims to evaluate the performance of QSSA for solving global optimization and to compare its performance with the original SSA and other meta-heuristic algorithms. In this section, 29 global benchmark optimization functions of CEC 2017 are considered. Different evaluation measurements are employed for this experiment. These measurements are standard deviation, mean and the p-values of the Wilcoxon rank sum. The aim of the second experiment in Section 4.2 is to further evaluate the performance of QSSA for optimizing the contrast of the color image. In this section, eight well-known color images taken from Matlab Demo Images are employed. These images are Hestain, Peppers, Fabric, Pears, Tissue, Tape, Westconcordaerial and Onion. Two evaluation measurements are used for evaluating the proposed QSSA for contrast enhancement of color images. These measurements are mean, square noise ratio (PSNR) and mean square error (MSE). In all the experiments, the results are reported on average 30 independent runs.

In addition, all the experimental results are executed on the same PC with same system specification; RAM $2 \mathrm{~GB}$ and Core i3 on OS Windows 7.

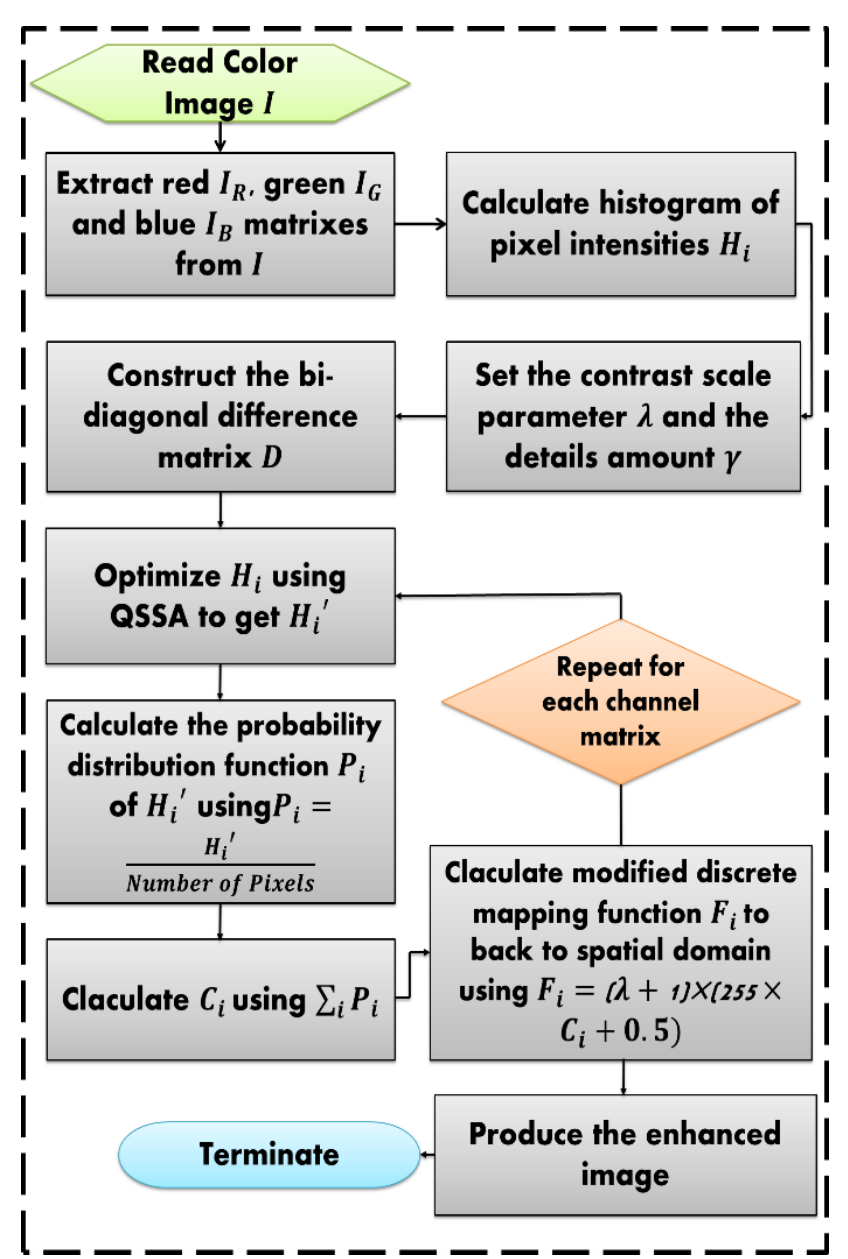

Figure. 1 QSSA flowchart for contrast enhancement of the color images

\subsection{QSSA for global optimization problem}

In this subsection, the performance of the proposed QSSA is evaluated using one of very recent benchmark functions called CEC 2017, where more complex problems different from previous competition benchmark functions are included. The mathematical formula with the properties of each benchmark function, the authors refer to [23]. The adopted benchmark functions are divided into two categories. The first category, namely the unimodal test function has no local optimum and only one global optimum. The convergence rate with the exploitation capability of an algorithm can be benchmarked using unimodal test function, as the entire search space favours the global optimum. The second category, namely the multimodal test function has many local optima. Thus it is suitable for evaluating the performance of an algorithm regarding the exploration capability and the ability to avoid the local optimum. Moreover, the 
multimodal test function is considered more challenging than the unimodal test function.

Table 1 shows the mean and standard deviation of 29 benchmark functions obtained from QSSA, SSA, particle swarm optimization (PSO) [24], bat algorithm (BA) [25], quantum particle swarm (QPSO) [18] and quantum artificial bee colony (QABC) [20].

PSO is one of widely used population-based stochastic algorithm, which simulates the social behavior of bird flocks. Unlike evolutionary algorithms, its implementation is easy and is computationally inexpensive. Each particle (candidate solution) moves in a multidimensional search space according to the knowledge achieved by interpersonal and social communication between the other particles [24]. QPSO is a modified version of PSO, where the wave function of quantum computing is used in the optimization process of PSO [18]. BA is another well-known meta-heuristic algorithm, which inspired by the echolocation behavior of microbats [25]. Each bat with a predefined velocity and position, flies in the search space to find its prey. The search is identified based on the local random walk. QABC is a quantum version of $\mathrm{ABC}$. The main inspiration of $\mathrm{ABC}$ came from the foraging behavior of honey bees to find the food source. In $\mathrm{ABC}$, the colony consists of three groups; scouts, onlookers and employed bees. Each one of them has a different role in the colony. QABC uses the principles of quantum physics to build a new update equation. This strategy can find a good balance between the exploration and the exploitation to obtain better results [20].

The parameter settings for QABC; the colony size equals to 10 , the number of food sources equals to 5 and the number of limit trials equals to 5 , while for BA; minimum Frequency equals to 0 , maximum Frequency equals to $2, A$ equals to 0.5 and $r$ equals to 0.5 , for PSO and QPSO; the inertial weight equals to 1 , the inertia weight damping ratio equals to 0.9 , personal learning coefficient equals to 1.5 and global learning coefficient equals to 2.0. It should be mentioned that in all experiments, the maximum number of iteration set to 500 with population size equals to 50. Moreover, all the algorithms start with the same initial positions, and use the same fitness function. Thus, it can be almost a fair comparison. The best results are underlined in this table. As it can be observed from this table, the proposed QSSA is superior, as it obtained the best results in most cases. Moreover, it can be observed that QPSO is in second place and SSA is in third place.
Table 1. QSSA vs. other meta-heuristic optimization algorithms in terms of mean

\begin{tabular}{|c|c|c|c|c|}
\hline & \multicolumn{2}{|c|}{ QSSA } & \multicolumn{2}{|c|}{ SSA [3] } \\
\hline & Mean & SD & Mean & SD \\
\hline F1 & $2.8 \times 10^{9}$ & $1.7 \times 10^{9}$ & $3.3 \times 10^{9}$ & $6.4 \times 10^{8}$ \\
\hline F2 & $2.1 \times 10^{5}$ & $3.2 \times 10^{5}$ & $\underline{4.8 \times 10^{4}}$ & $8.3 \times 10^{3}$ \\
\hline F3 & $8.4 \times 10^{3}$ & $1.1 \times 10^{3}$ & $1.1 \times 10^{4}$ & $1.1 \times 10^{3}$ \\
\hline F4 & $2.1 \times 10^{2}$ & $\overline{7.6 \times 10^{0}}$ & $2.2 \times 10^{2}$ & $3.4 \times 10^{0}$ \\
\hline F5 & $\overline{7.2 \times 10^{1}}$ & $\overline{5.4 \times 10^{-1}}$ & $\underline{7.1 \times 10^{1}}$ & $\underline{3.9 \times 10^{-1}}$ \\
\hline F6 & $4.8 \times 10^{2}$ & $3.7 \times 10^{1}$ & $\overline{5.2 \times 10^{2}}$ & $\overline{1.0 \times 10^{1}}$ \\
\hline F7 & $2.6 \times 10^{2}$ & $7.6 \times 10^{0}$ & $2.7 \times 10^{2}$ & $7.4 \times 10^{0}$ \\
\hline F8 & $\overline{1.2 \times 10^{4}}$ & $1.1 \times 10^{3}$ & $\underline{1.1 \times 10^{4}}$ & $\underline{6.8 \times 10^{2}}$ \\
\hline F9 & $3.4 \times 10^{3}$ & $1.0 \times 10^{2}$ & $3.3 \times 10^{3}$ & $8.0 \times 10^{1}$ \\
\hline F10 & $3.8 \times 10^{4}$ & $\underline{6.7 \times 10^{3}}$ & $4.6 \times 10^{4}$ & $7.6 \times 10^{3}$ \\
\hline F11 & $\overline{1.5 \times 10^{9}}$ & $1.5 \times 10^{9}$ & $1.9 \times 10^{9}$ & $1.6 \times 10^{9}$ \\
\hline F12 & $4.4 \times 10^{9}$ & $5.6 \times 10^{8}$ & $5.4 \times 10^{9}$ & $5.0 \times 10^{8}$ \\
\hline F13 & $7.5 \times 10^{6}$ & $3.1 \times 10^{6}$ & $1.4 \times 10^{7}$ & $5.1 \times 10^{6}$ \\
\hline F14 & $1.9 \times 10^{9}$ & $3.5 \times 10^{8}$ & $2.1 \times 10^{9}$ & $3.6 \times 10^{8}$ \\
\hline F15 & $2.1 \times 10^{3}$ & $\overline{2.5 \times 10^{2}}$ & $2.3 \times 10^{3}$ & $3.1 \times 10^{2}$ \\
\hline F16 & $2.3 \times 10^{5}$ & $1.9 \times 10^{5}$ & $5.2 \times 10^{5}$ & $6.3 \times 10^{5}$ \\
\hline F17 & $1.7 \times 10^{7}$ & $6.5 \times 10^{6}$ & $3.8 \times 10^{7}$ & $1.4 \times 10^{7}$ \\
\hline F18 & $1.6 \times 10^{9}$ & $2.6 \times 10^{8}$ & $2.1 \times 10^{9}$ & $4.8 \times 10^{8}$ \\
\hline F19 & $\overline{8.6 \times 10^{2}}$ & $\overline{4.7 \times 10^{1}}$ & $8.7 \times 10^{2}$ & $3.8 \times 10^{1}$ \\
\hline F20 & $\overline{4.5 \times 10^{2}}$ & $\overline{1.4 \times 10^{1}}$ & $4.8 \times 10^{2}$ & $1.3 \times 10^{1}$ \\
\hline F21 & $3.6 \times 10^{3}$ & $9.2 \times 10^{1}$ & $3.7 \times 10^{3}$ & $8.7 \times 10^{1}$ \\
\hline F22 & $6.9 \times 10^{2}$ & $4.0 \times 10^{1}$ & $6.4 \times 10^{2}$ & $3.2 \times 10^{1}$ \\
\hline F23 & $1.0 \times 10^{3}$ & $6.2 \times 10^{1}$ & $8.8 \times 10^{2}$ & $5.6 \times 10^{1}$ \\
\hline F24 & $3.4 \times 10^{3}$ & $3.8 \times 10^{2}$ & $3.8 \times 10^{3}$ & $1.4 \times 10^{2}$ \\
\hline F25 & $5.6 \times 10^{3}$ & $4.1 \times 10^{2}$ & $6.2 \times 10^{3}$ & $2.8 \times 10^{2}$ \\
\hline F26 & $3.1 \times 10^{2}$ & $5.8 \times 10^{-14}$ & $3.2 \times 10^{2}$ & $5.8 \times 10^{-14}$ \\
\hline F27 & $2.7 \times 10^{3}$ & $1.3 \times 10^{2}$ & $3.4 \times 10^{3}$ & $1.3 \times 10^{2}$ \\
\hline F28 & $\overline{2.9 \times 10^{4}}$ & $\overline{1.7 \times 10^{4}}$ & $1.4 \times 10^{5}$ & $1.1 \times 10^{5}$ \\
\hline \multirow[t]{3}{*}{ F29 } & $3.0 \times 10^{9}$ & $6.2 \times 10^{8}$ & $3.2 \times 10^{9}$ & $4.0 \times 10^{8}$ \\
\hline & \multicolumn{2}{|c|}{ QPSO [18] } & \multicolumn{2}{|c|}{ PSO [24] } \\
\hline & Mean & Mean & Mean & SD \\
\hline F1 & $3.1 \times 10^{9}$ & $6.5 \times 10^{9}$ & $6.5 \times 10^{9}$ & $1.7 \times 10^{9}$ \\
\hline F2 & $1.7 \times 10^{7}$ & $3.6 \times 10^{5}$ & $3.6 \times 10^{5}$ & $2.8 \times 10^{9}$ \\
\hline F3 & $8.9 \times 10^{3}$ & $1.9 \times 10^{4}$ & $1.9 \times 10^{4}$ & $9.7 \times 10^{2}$ \\
\hline F4 & $2.3 \times 10^{2}$ & $2.9 \times 10^{2}$ & $2.9 \times 10^{2}$ & $5.5 \times 10^{0}$ \\
\hline F5 & $7.2 \times 10^{1}$ & $7.7 \times 10^{1}$ & $7.7 \times 10^{1}$ & $0.6 \times 10^{0}$ \\
\hline F6 & $7.5 \times 10^{2}$ & $1.3 \times 10^{3}$ & $1.3 \times 10^{3}$ & $\underline{8.1 \times 10^{0}}$ \\
\hline F7 & $2.7 \times 10^{2}$ & $3.6 \times 10^{2}$ & $3.6 \times 10^{2}$ & $\overline{5.3 \times 10^{0}}$ \\
\hline F8 & $1.4 \times 10^{4}$ & $2.0 \times 10^{4}$ & $2.0 \times 10^{4}$ & $1.4 \times 10^{3}$ \\
\hline F9 & $3.5 \times 10^{3}$ & $3.8 \times 10^{3}$ & $3.8 \times 10^{3}$ & $7.2 \times 10^{1}$ \\
\hline F10 & $5.3 \times 10^{4}$ & $5.7 \times 10^{4}$ & $5.7 \times 10^{4}$ & $1.7 \times 10^{8}$ \\
\hline F11 & $1.1 \times 10^{9}$ & $3.4 \times 10^{9}$ & $3.4 \times 10^{9}$ & $1.5 \times 10^{9}$ \\
\hline F12 & $2.3 \times 10^{9}$ & $1.0 \times 10^{9}$ & $1.0 \times 10^{9}$ & $3.7 \times 10^{8}$ \\
\hline F13 & $1.3 \times 10^{7}$ & $8.4 \times 10^{7}$ & $8.4 \times 10^{7}$ & $3.1 \times 10^{7}$ \\
\hline F14 & $2.3 \times 10^{9}$ & $6.1 \times 10^{9}$ & $6.1 \times 10^{9}$ & $2.2 \times 10^{8}$ \\
\hline F15 & $1.8 \times 10^{3}$ & $4.4 \times 10^{3}$ & $4.4 \times 10^{3}$ & $2.8 \times 10^{2}$ \\
\hline F16 & $\overline{4.3 \times 10^{5}}$ & $7.8 \times 10^{6}$ & $7.8 \times 10^{6}$ & $3.3 \times 10^{6}$ \\
\hline F17 & $3.1 \times 10^{7}$ & $2.2 \times 10^{8}$ & $2.2 \times 10^{8}$ & $2.8 \times 10^{7}$ \\
\hline F18 & $8.6 \times 10^{9}$ & $4.3 \times 10^{9}$ & $4.3 \times 10^{9}$ & $5.4 \times 10^{8}$ \\
\hline F19 & $8.7 \times 10^{2}$ & $1.0 \times 10^{3}$ & $1.0 \times 10^{3}$ & $5.1 \times 10^{1}$ \\
\hline F20 & $4.3 \times 10^{2}$ & $5.2 \times 10^{2}$ & $5.2 \times 10^{2}$ & $3.3 \times 10^{1}$ \\
\hline F21 & $\overline{3.8 \times 10^{3}}$ & $3.9 \times 10^{3}$ & $3.9 \times 10^{3}$ & $9.9 \times 10^{1}$ \\
\hline F22 & $\underline{5.4 \times 10^{2}}$ & $9.2 \times 10^{2}$ & $9.2 \times 10^{2}$ & $4.9 \times 10^{1}$ \\
\hline F23 & $8.5 \times 10^{2}$ & $1.2 \times 10^{3}$ & $1.2 \times 10^{3}$ & $1.1 \times 10^{2}$ \\
\hline
\end{tabular}

DOI: $10.22266 /$ ijies2019.1231.22 


\begin{tabular}{|c|c|c|c|c|}
\hline F24 & $4.6 \times 10^{3}$ & $1.4 \times 10^{4}$ & $1.4 \times 10^{4}$ & $2.5 \times 10^{2}$ \\
\hline F25 & $6.3 \times 10^{3}$ & $1.0 \times 10^{4}$ & $1.0 \times 10^{4}$ & $\overline{4.5 \times 10^{2}}$ \\
\hline F26 & $8.1 \times 10^{2}$ & $2.0 \times 10^{3}$ & $2.0 \times 10^{3}$ & $6.5 \times 10^{1}$ \\
\hline F27 & $3.8 \times 10^{3}$ & $6.6 \times 10^{3}$ & $6.6 \times 10^{3}$ & $4.1 \times 10^{2}$ \\
\hline F28 & $1.6 \times 10^{4}$ & $1.7 \times 10^{6}$ & $1.7 \times 10^{6}$ & $2.3 \times 10^{5}$ \\
\hline \multirow[t]{3}{*}{ F29 } & $3.3 \times 10^{9}$ & $6.6 \times 10^{9}$ & $6.6 \times 10^{9}$ & $4.7 \times 10^{8}$ \\
\hline & \multicolumn{2}{|c|}{ QABC [20] } & \multicolumn{2}{|c|}{ BA [25] } \\
\hline & Mean & Mean & Mean & SD \\
\hline F1 & $2.9 \times 10^{9}$ & $2.9 \times 10^{9}$ & $6.5 \times 10^{9}$ & $1.6 \times 10^{-5}$ \\
\hline F2 & $8.1 \times 10^{9}$ & $8.1 \times 10^{9}$ & $1.1 \times 10^{7}$ & $3.8 \times 10^{-9}$ \\
\hline F3 & $1.5 \times 10^{4}$ & $1.5 \times 10^{4}$ & $1.9 \times 10^{4}$ & $7.4 \times 10^{-12}$ \\
\hline F4 & $2.3 \times 10^{2}$ & $2.3 \times 10^{2}$ & $2.9 \times 10^{2}$ & $5.8 \times 10^{-14}$ \\
\hline F5 & $7.3 \times 10^{1}$ & $7.3 \times 10^{1}$ & $7.7 \times 10^{1}$ & $1.5 \times 10^{-14}$ \\
\hline F6 & $4.3 \times 10^{2}$ & $4.3 \times 10^{2}$ & $1.3 \times 10^{3}$ & $0.0 \times 10^{0}$ \\
\hline F7 & $2.8 \times 10^{2}$ & $\overline{2.8 \times 10^{2}}$ & $3.6 \times 10^{2}$ & $2.9 \times 10^{-13}$ \\
\hline F8 & $1.1 \times 10^{4}$ & $1.1 \times 10^{4}$ & $2.1 \times 10^{4}$ & $3.7 \times 10^{-12}$ \\
\hline F9 & $3.6 \times 10^{3}$ & $3.6 \times 10^{3}$ & $3.8 \times 10^{3}$ & $5.0 \times 10^{1}$ \\
\hline F10 & $3.6 \times 10^{7}$ & $3.6 \times 10^{7}$ & $5.7 \times 10^{4}$ & $2.2 \times 10^{-11}$ \\
\hline F11 & $2.5 \times 10^{9}$ & $2.5 \times 10^{9}$ & $3.4 \times 10^{9}$ & $2.3 \times 10^{-5}$ \\
\hline F12 & $6.0 \times 10^{9}$ & $6.0 \times 10^{9}$ & $1.1 \times 10^{9}$ & $1.9 \times 10^{-6}$ \\
\hline F13 & $5.2 \times 10^{7}$ & $5.2 \times 10^{7}$ & $8.4 \times 10^{7}$ & $4.6 \times 10^{-8}$ \\
\hline F14 & $4.0 \times 10^{9}$ & $4.0 \times 10^{9}$ & $6.1 \times 10^{9}$ & $2.9 \times 10^{-6}$ \\
\hline F15 & $3.5 \times 10^{3}$ & $3.5 \times 10^{3}$ & $4.4 \times 10^{3}$ & $1.9 \times 10^{-12}$ \\
\hline F16 & $5.0 \times 10^{6}$ & $5.0 \times 10^{6}$ & $7.8 \times 10^{6}$ & $2.8 \times 10^{-9}$ \\
\hline F17 & $5.2 \times 10^{7}$ & $5.2 \times 10^{7}$ & $2.4 \times 10^{8}$ & $9.1 \times 10^{-8}$ \\
\hline F18 & $3.4 \times 10^{9}$ & $3.4 \times 10^{9}$ & $4.3 \times 10^{9}$ & $2.4 \times 10^{-6}$ \\
\hline F19 & $9.5 \times 10^{2}$ & $9.5 \times 10^{2}$ & $1.0 \times 10^{3}$ & $2.6 \times 10^{1}$ \\
\hline F20 & $5.4 \times 10^{2}$ & $5.4 \times 10^{2}$ & $5.2 \times 10^{2}$ & $2.3 \times 10^{-13}$ \\
\hline F21 & $3.9 \times 10^{3}$ & $3.9 \times 10^{3}$ & $3.9 \times 10^{3}$ & $3.2 \times 10^{1}$ \\
\hline F22 & $8.4 \times 10^{2}$ & $8.4 \times 10^{2}$ & $7.7 \times 10^{2}$ & $3.7 \times 10^{1}$ \\
\hline F23 & $1.4 \times 10^{3}$ & $1.4 \times 10^{3}$ & $1.2 \times 10^{3}$ & $0.0 \times 10^{0}$ \\
\hline F24 & $3.4 \times 10^{3}$ & $\underline{3.4 \times 10^{3}}$ & $1.4 \times 10^{4}$ & $7.4 \times 10^{-12}$ \\
\hline F25 & $6.2 \times 10^{3}$ & $6.2 \times 10^{3}$ & $1.0 \times 10^{4}$ & $1.9 \times 10^{-12}$ \\
\hline F26 & $1.4 \times 10^{3}$ & $1.4 \times 10^{3}$ & $2.0 \times 10^{3}$ & $6.9 \times 10^{-13}$ \\
\hline F27 & $3.6 \times 10^{3}$ & $3.6 \times 10^{3}$ & $6.6 \times 10^{3}$ & $2.8 \times 10^{-12}$ \\
\hline F28 & $4.8 \times 10^{5}$ & $4.8 \times 10^{5}$ & $1.7 \times 10^{6}$ & $1.2 \times 10^{-9}$ \\
\hline F29 & $5.8 \times 10^{9}$ & $5.8 \times 10^{9}$ & $6.6 \times 10^{9}$ & $3.9 \times 10^{-6}$ \\
\hline
\end{tabular}

As the authors of QPSO mentioned in [18], QPSO like PSO is sensitive to its parameters. Thus, they set the control parameters based on trial. In this paper, we used the default parameters setting of all the competitors' algorithms, as we mainly focused on improving the performance of SSA. Also, it can be observed that BA obtained the worst results. The reason behind this, PSO and BA unlike the recent swarm algorithms such as SSA has many parameters need to be tuned so that the algorithm can optimally find the global optima. These parameters can have significantly effect on the algorithm performance. Also, the reason behind the low performance of BA may be due to improper balancing between exploration and exploitation. In this work, we are mainly focused on boosting the performance of only SSA through embedding the principles of quantum computing in the optimization process of SSA.
Table 2. QSSA vs. other meta-heuristic optimization algorithms in terms of p-values of Wilcoxon ranksum test

\begin{tabular}{|l|c|}
\hline & $<\mathbf{0 . 0 5}$ \\
\hline QPSO [18] & $\begin{array}{c}\text { F1, F2, F4-F17, F19- } \\
\text { F29 }\end{array}$ \\
\hline QABC [20] & F1-F29 \\
\hline SSA [3] & F1-F4, F6-F8,F10-F29 \\
\hline PSO [24] & F1-F29 \\
\hline BA [25] & F1-F29 \\
\hline
\end{tabular}

Through this strategy, the proposed QSSA can significantly explore the most promising area in the search space and thus it can find a good balance between the exploration and the exploitation.

Table 2 summarizes the reported $\mathrm{p}$-values of the Wilcoxon ranksum test of two pairs; QSSA vs. SSA, QSSA vs. PSO, QSSA vs. BA, QSSA vs. QABC and QSSA vs. QPSO. As it can be seen QSSA is statistically significant. This is due to the most of the obtained results are less than 0.05. Also, it can be observed that QSSA outperforms BA, PSO and $\mathrm{QABC}$, as the reported values from all functions are less than 0.5. From all the obtained results, it can be observed that the proposed QSSA is competitive algorithm and can find better solutions compared with the other algorithms. Moreover, it can be indicated that QSSA is robust in solving both unimodal and multimodal optimization problems. Next, further evaluation of the proposed QSSA will be discussed.

\subsection{QSSA for contrast enhancement problem}

In this section, the modified version of SSA is proposed to solve contrast enhancement problem. Eight well-known images are considered to perform image enhancement experiment. The results of the proposed quantum salp swarm based contrast image enhancement (QSSA-IE) are compared with salp swarm based contrast image enhancement (SSA-IE) and previously published algorithms. These algorithms are artificial bee colony based image enhancement (ABC-IE) [26] and particle swarm based image enhancement (PSO-IE) [27]. Several evaluation criteria are considered for evaluation purpose. These criteria are divided into subjective and objective criterion. The subjective criterion is the visual quality, the objective criterion are mean intensity of the image, Peak Signal-to-Noise Ratio (PSNR) and Mean Squared Error (MSE). The mathematical definitions of MSE and PSNR are defined in Eqs. (17) and (18), respectively. 


$$
\begin{gathered}
M S E=\frac{\sum_{M, N}[O(m, n)-E(m, n)]^{2}}{M \times N} \\
P S N R=20 \log _{10} \frac{255}{\sqrt{M S E}}
\end{gathered}
$$

where $O$ is the original image, $E$ is the enhanced image, $N$ and $M$ are the number of rows and columns in the image.

Table 3 compares the mean intensity of the image from the original image, QSSA-IE, SSA-IE, PSO-IE and ABC-IE. Mean intensity of the image is calculated through taking the average of the intensity of all pixels in the image. As it can be observed, the proposed QSSA-IE can improve the image quality, as it obtains the highest mean intensity in most cases. Also, it can be observed that the results of QSSA-IE are better than SSA-IE.

Table 2. Mean intensity values obtained from original image and modified metaheuristic optimization

\begin{tabular}{|c|c|c|c|c|c|}
\hline & Original & $\begin{array}{c}\text { SSA- QSSA- } \\
\text { IE }\end{array}$ & $\begin{array}{c}\text { ABC- } \\
\text { IE }\end{array}$ & $\begin{array}{c}\text { PSO- } \\
\text { IE[27] }\end{array}$ \\
\hline Hestain & 175 & 214 & 215 & $\underline{220}$ & 218 \\
\hline Peppers & 82 & $\underline{115}$ & 111 & 102 & 111 \\
\hline Fabric & 90 & 114 & $\underline{119}$ & 116 & 114 \\
\hline Pears & 130 & 139 & $\underline{143}$ & 141 & 142 \\
\hline Tissue & 180 & 206 & $\underline{209}$ & 203 & 208 \\
\hline Tape & 66 & 124 & $\underline{128}$ & $\underline{128}$ & 123 \\
\hline $\begin{array}{c}\text { Westco } \\
\text { ncordae } \\
\text { rial }\end{array}$ & 140 & 190 & 193 & $\underline{195}$ & 187 \\
\hline Onion & 92 & 171 & 170 & 168 & $\underline{178}$ \\
\hline
\end{tabular}

Table 3. MSE obtained from QSSA-IE and other metaheuristic optimization algorithms

\begin{tabular}{|c|c|c|c|c|}
\hline & $\begin{array}{c}\text { SSA- } \\
\text { IE }\end{array}$ & $\begin{array}{c}\text { QSSA- } \\
\text { IE }\end{array}$ & $\begin{array}{c}\text { ABC- } \\
\text { IE[26] }\end{array}$ & $\begin{array}{c}\text { PSO- } \\
\text { IE[27] }\end{array}$ \\
\hline Hestain & 228.60 & $\underline{226.34}$ & 228.00 & 229.00 \\
\hline Peppers & 227.00 & $\underline{218.00}$ & 233.00 & 231.00 \\
\hline Fabric & 231.00 & $\underline{206.00}$ & 227.00 & 237.00 \\
\hline Pears & 85.45 & $\underline{29.19}$ & 111.00 & 133.00 \\
\hline Tissue & 202.00 & 196.00 & $\underline{188.00}$ & 200.00 \\
\hline Tape & 247.00 & $\underline{232.00}$ & 250.00 & 250.00 \\
\hline $\begin{array}{c}\text { Westconc } \\
\text { ordaerial }\end{array}$ & 253.00 & $\underline{251.00}$ & 253.00 & 252.00 \\
\hline Onion & 237.00 & $\underline{233.00}$ & 237.00 & 238.00 \\
\hline
\end{tabular}

Table 4. PSNR obtained from QSSA-IE and other metaheuristic optimization algorithms

\begin{tabular}{|c|c|c|c|c|}
\hline & $\begin{array}{c}\text { SSA- } \\
\text { IE }\end{array}$ & $\begin{array}{c}\text { QSSA- } \\
\text { IE }\end{array}$ & $\begin{array}{c}\text { ABC- } \\
\text { IE[26] }\end{array}$ & $\begin{array}{c}\text { PSO- } \\
\text { IE[27] }\end{array}$ \\
\hline Hestain & 24.54 & $\underline{24.58}$ & 24.55 & 24.53 \\
\hline Peppers & 24.56 & $\underline{24.74}$ & 24.45 & 24.50 \\
\hline Fabric & 24.49 & $\underline{24.99}$ & 24.57 & 24.38 \\
\hline Pears & 28.81 & $\underline{33.48}$ & 27.69 & 26.89 \\
\hline Tissue & 25.08 & $\underline{25.20}$ & $\underline{25.29}$ & 25.12 \\
\hline Tape & 24.20 & $\underline{24.48}$ & 24.16 & 24.15 \\
\hline $\begin{array}{c}\text { Westconc } \\
\text { ordaerial }\end{array}$ & 24.13 & $\underline{24.45}$ & 24.10 & 24.12 \\
\hline Onion & 24.38 & $\underline{24.46}$ & 24.39 & 24.36 \\
\hline
\end{tabular}

Table 4 compares MSE obtained from QSSA-IE, SSA-IE, ABC-IE and PSO-IE. Mean square error (MSE) is calculated through subtracting the intensity of original image from the intensity of enhanced image, and then taking the average. As it can be observed, there is a decrease in MSE values of the adopted images obtained from QSSA-IE over the other algorithms. This low MSE values can indicate an improvement in the objective quality of the images.

Table 5 compares PSNR values obtained from QSSA-IE, SSA-IE, ABC-IE and PSO-IE. Peak square noise ratio (PSNR) is the proportion between the maximum possible value of the transmission and MSE (the power of the corrupting noise), where the larger the value, the less the image corruption.

As it can be observed, the larger values obtained when QSSA-IE is used over the other image enhancement algorithms. Despite mean intensity, PSNR and MSE value are quantitative measurements of the image quality; they cannot be the same as human eye [28]. This is due to the sensitivity to the errors isn't absolute. Thus, the image may not be considered well enhanced in case of trivial distortion.

Figs. 2 and 3 show the original hestain and peppers images and with their enhanced images versions from QSSA-IE, SSA-IE, ABC-IE and PSOIE. As it can be observed, the enhanced image using QSSA-IE can significantly enhance the contrast of the image as well as preserve the details of the image. Furthermore, it can efficiently save the color of the images. Also, the results show that QSSA-IE provides outstanding results compared with the other image enhancement algorithms. 


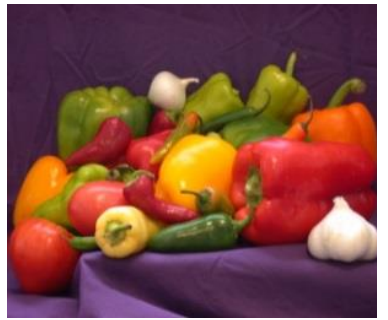

(a)

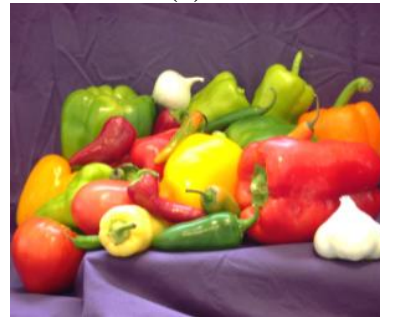

(c)

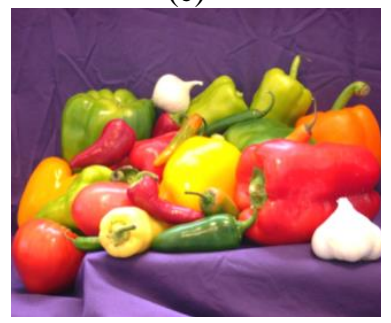

(e)

Figure. 2 Simulation results: (a) the original Peppers

Image, (b) contrast enhancement by QSSA-IE, (c) contrast enhancement by PSO-IE, (d) contrast enhancement by SSA-IE and (e) contrast enhancement by ABC-IE

\section{Conclusions}

This study introduces a new hybrid approach based on SSA and quantum computing, where the quantum operators and the interference are embedded in the optimization process of SSA. In QSSA, the positioning of salps is modified according to quantum mechanics to obtain a good balance between exploitation and exploration. The proposed hybrid approach is applied on two optimization problems; global optimization and contrast enhancement optimization problems, where 29 global high-dimensional benchmark functions of CEC 2017 and 8 benchmark images of Matlab Demo Images are used. The experimental results showed that the embedding the principles of quantum computing in the optimization process of SSA can explore the most promising area in the search space. Thus, this strategy can significantly boost the performance of SSA. Also, the results on CEC 2017 showed that the proposed QSSA is distinctly superior to SSA, PSO, BA, QPSO and QABC in terms of mean, standard deviation and $p$ values of the Wilcoxon ranksum test. Additionally, the experimental results on 8 benchmark images

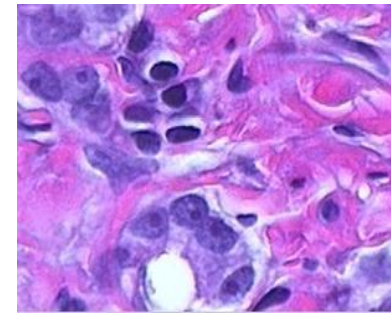

(a)

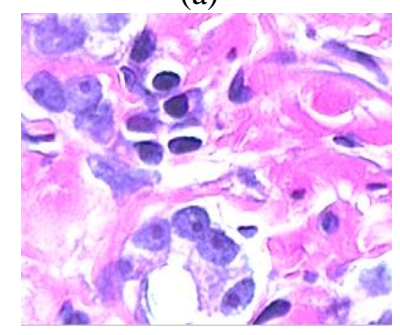

(c)

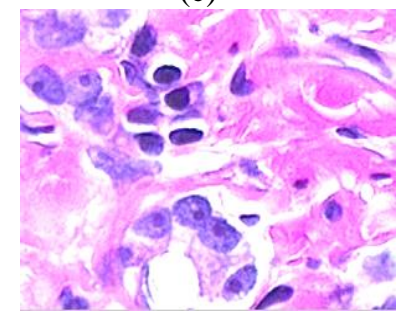

(e)

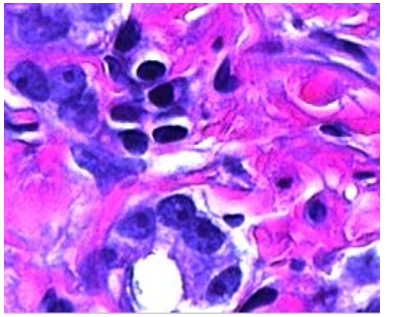

(b)

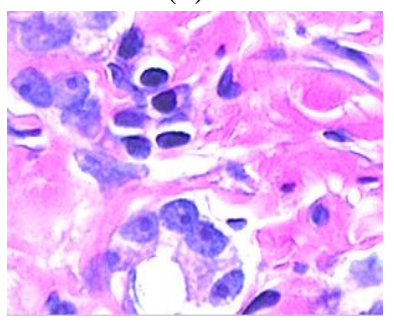

(d)
Figure. 3 Simulation results: (a) the original Hestain

Image, (b) contrast enhancement by QSSA-IE, (c) contrast enhancement by PSO-IE, (d) contrast enhancement by SSA-IE and (e) contrast enhancement by ABC-IE

revealed that the proposed QSSA can significantly enhance the contrast of the image as well as preserve the details of the image. Moreover, the results demonstrated that the proposed image enhancement based QSSA is robust and exhibits better performance than image enhancement based on PSO and $\mathrm{ABC}$ in terms of both qualitative and quantitative measurements.

For future studies, the proposed QSSA will be employed to solve other QSSA for contrast enhancement problem real-world optimization problems.

\section{References}

[1] X. Yang, "Review of Meta-heuristics and Generalized Evolutionary Walk Algorithm", International Journal of Bio-Inspired Computation, Vol.3, No.2, pp.77-84, 2011.

[2] Gogna and A. Tayal, "Metaheuristics: Review and Application", Journal of Experimental \& Theoretical Artificial Intelligence, Vol.25, No.4, pp.503-526, 2013.

[3] S. Mirjalili, A. Gandomi, Z. Mirjalili, S. Saremi, H. Faris, and S. Mirjalili, "Salp Swarm Algorithm: A Bio-Inspired Optimizer for 
Engineering Design Problems", Advances in Engineering Software, Vol.114, pp.163-191, 2017.

[4] P. Benioff, "The Computer as A Physical System: Microscopic Quantum Mechanical Hamiltonian Model of Computers as Represented by Turing Machines", Journal of Statistical Physics, Vol.22, No.5, pp.563-591, 1980.

[5] K. Han and J. Kim, "Quantum-Inspired Evolutionary Algorithms With A New Termination Criterion, $\mathrm{H}$ Gate, and Two Phase Scheme", IEEE Transactions on Evolutionary Computation, Vol.8, No.2, pp.156-169, 2004.

[6] A. Layeb and D. Saidouni, "A New Quantum Evolutionary Local Search Algorithm For Max 3-sat Problem", In: Proc. of International Workshop on Hybrid Artificial Intelligence Systems, pp.172-179, 2008.

[7] S. Tao, D. Liu, and A. Tang, "Bridge Critical State Search by Using Quantum Genetic Firefly Algorithm", Shock and Vibration, Vol.2019, pp.1-10, 2019.

[8] A. Layeb, "Hybrid Quantum Scatter Search Algorithm for Combinatorial Optimization Problems", Annals. Computer Science Series, Vol.8, No.2, pp.227-244, 2010.

[9] X. Su, W. Fang, Q. Shen, and X. Hao, "An Image Enhancement Method Using the Quantum Behaved Particle Swarm Optimization with an Adaptive Strategy", Mathematical Problems in Engineering, Vol.2013, pp.1-14, 2013.

[10] M. Kanmani and V. Narsimhan, "An Image Contrast Enhancement Algorithm for Grayscale Images Using Particle Swarm Optimization", Multimedia Tools and Applications, Vol.77, No.18, pp. 23371-23387, 2018.

[11] M. Aladem, S. Baek, and S. Rawashdeh, "Evaluation of Image Enhancement Techniques for Vision Based Navigation under Low Illumination", Journal of Robotics, Vol.2019, pp.1-15, 2019.

[12] W. Wang, N. Sun, and M. Ng, "A Variational Gamma Correction Model for Image Contrast Enhancement", Inverse Problems \& Imaging, Vol.13, No.3, pp.461-478, 2019.

[13] T. Arici, S. Dikbas, and Y. Altunbasak, "A Histogram Modification Framework and Its Application for Image Contrast Enhancement", IEEE Transactions on Image Processing, Vol.18, No.9, pp.1921-1935, 2009.

[14] N. Li and J. Zhang, "Automatic Image Enhancement by Learning Adaptive Patch Selection", Frontiers of Information
Technology \& Electronic Engineering, Vol.20, No.2, pp.206-221, 2019.

[15] G. Sayed, G. Khoriba, and M. Haggag, "A Novel Chaotic Salp Swarm Algorithm for Global Optimization and Feature selection", Applied Intelligence, Vol.48, No.10, pp. 34623481, 2018.

[16] W. He and Y. Shi, "Multiobjective Construction Optimization Model Based on Quantum Genetic Algorithm", Advances in Civil Engineering, Vol.2019, pp.1-8, 2019.

[17] M. Ying, "Quantum Computation, Quantum Theory and AI", Artificial Intelligence, Vol.174, No.1, pp.162-176, 2010.

[18] J. Sun, B. Feng, and W. Xu, "Particle Swarm Optimization with Particles Having Quantum Behavior", In: Proc. of Congress on Evolutionary Computation, pp.325-331, 2004.

[19] R. Orús, S. Mugel, and E. Lizaso, "Quantum Computing for Finance: Overview and Prospects", Reviews in Physics, Vol.4, pp.1-12, 2019.

[20] H. Saadoon, "Quantum Artificial Bee Colony Algorithm for Numerical Function Optimization", International Journal of Computer Applications, Vol.93, No.3, pp.28-33, 2014.

[21] J. Liu, W. Xu, and J. Sun, "Quantum Behaved Particle Swarm Optimization with Mutation Operator", In: Proc. of IEEE International Conference on Tools with Artificial Intelligence, pp.240-244, 2005.

[22] L. Coelho, "A Quantum Particle Swarm Optimizer with Chaotic Mutation Operator", Chaos, Solitons \& Fractals, Vol.37, No.5, pp.1409-1418, 2008.

[23] A. LaTorre and J. Peña, "A Comparison of Three Large-Scale Global Optimizers on The CEC 2017 Single Objective Real Parameter Numerical Optimization Benchmark", In: Proc. of IEEE Congress on Evolutionary Computation, pp.1063-1070, 2017.

[24] J. Kennedy and R. Eberhart. "Particle Swarm Optimization". In: Proc. of IEEE International Conference on Neural Networks, pp. 19421948, 1995.

[25] X. Yang, "A new metaheuristic bat-inspired algorithm", In: Proc. of Nature-Inspired Cooperative Strategies for Optimization, pp.6574, 2010.

[26] J. Chen, W. Yu, J. Tian, and L. Chen. "Adaptive Image Contrast Enhancement Using Artificial Bee Colony Optimization", In: Proc. of IEEE International Conference on Image Processing, pp. 3220-3224, 2017. 
[27] A. Seema and G. Bansal, "Image Contrast Enhancement Approach Using Differential Evolution and Particle Swarm Optimization", International Research Journal of Engineering and Technology, Vol.4, No.8, pp.1134-1138, 2017.

[28] Y. Zhou, C. Shi, B. Lai, and G. Jimenez, "Contrast Enhancement of Medical Images Using a New Version of The World Cup Optimization Algorithm", Quantitative Imaging in Medicine and Surgery, Vol.9, No.9, pp.15281547, 2019. 